\title{
PHOTOLUMINESCENCE STUDIES OF GaN AND AlGaN LAYERS UNDER HYDROSTATIC PRESSURE
}

\author{
C. Wetzel, W. Walukiewicz, E.E. Haller, H. Amano*, and I. Akasaki* \\ Lawrence Berkeley Laboratory, Berkeley, CA 94720, USA \\ *Dept. of Electrical and Electronic Engineering, Meijo University, Nagoya, Japan
}

\begin{abstract}
Wide bandgap GaN very often shows a high electron concentration. Although several impurities such as $\mathrm{O}$ and $\mathrm{Si}$ have been identified, the concentration is not high enough to account for the number of free carriers. As a consequence native defects namely the nitrogen vacancies are widely considered to be present at high densities. Several calculations predict different energy levels of this strongly localized defect. We present photoluminescence experiments of wurtzite $\mathrm{GaN}$ and $\mathrm{AlGaN}$ layers under large hydrostatic pressure to search for localized defects within the questionable energy range of 3.0 to $3.8 \mathrm{eV}$ above the valence band edge.
\end{abstract}

\section{Introduction}

The growing interest in GaN and the wide bandgap III-V nitrides is driven by the recent demonstration of UV and blue light emitting devices [1]. This success has been achieved by a continuous basic research effort over the last view decades. Although promising device performances have been obtained, a great amount of details of the electronic and defect structure are still not understood. As observed in GaN material grown by many different techniques, it is very often highly n-type conducting in the range of $10^{18}-10^{20} \mathrm{~cm}^{-3}$ [2]. However no impurities could be observed or identified by any technique at that high concentration. Secondary ion mass spectroscopy (SIMS) results indeed reveal incorporation of $\mathrm{O}$ or $\mathrm{Si}$ and both of those elements should act as a donor in $\mathrm{GaN}$ [3,4], yet concentration typically ranges about two orders of magnitude below the observed carrier concentration in highly conducting material [5-8]. Based on the presently available data, impurities can not be the major source of dopands in GaN. Consequently native defects are considered to be present in very high concentrations.

Calculations by several groups predict the charge states and the energy levels of native defects [9-11]. At present only isolated defects rather then complexes have been considered, and among group III vacancies, group $\mathrm{V}$ vacancies and antisites the nitrogen vacancy $\mathrm{V}_{\mathrm{N}}$ is the best candidate to act as a donor. From several stoichiometry considerations this candidate has been in Defect and Impurity Engineered Semiconductors and Devices, Eds. S. Ashok, J. Chevallier, I. Akasaki, N.M. Johnson, and B.L. Sopori, Proc. Mat. Res. Soc. Symp. 378, 509-14 (1995). 
favored in many publications $[12,13]$. However no experimental evidence of the existence of this defect could be given so far. The model of Jenkins et al. [9] predict two levels $\mathrm{A}_{1}$ and $\mathrm{T}_{2}$ of the neutral $\mathrm{V}_{\mathrm{N}}$ to lie close to the conduction band (CB). $\mathrm{A}_{1}$ should be filled with two electrons and lie $\sim 0.2 \mathrm{eV}$ below $\mathrm{CB}$ whereas $\mathrm{T}_{2}$ should carry one out of six electrons and lie $0.6 \mathrm{eV}$ above the $\mathrm{CB}$. Being resonant with the $\mathrm{CB}$ the electron would auto-ionize, thermalize to the band edge and finally be bound in a hydrogenic state of the remaining fixed positive charge. Boguslawski et al. [10] predict both of those levels to lie within the $\mathrm{CB}, \mathrm{A}_{1}$ at $\mathrm{E}_{\mathrm{CB}}+0.8 \mathrm{eV}$, $\mathrm{T}_{2}$ at $\mathrm{E}_{\mathrm{CB}}+1.3 \mathrm{eV}$. In a third calculation by Neugebauer and Van de Walle [11] existence of the resonant level $\mathrm{T}_{2}$ has been confirmed, in contrast to the other predictions the $\mathrm{A}_{1}$ level should lie within the valence band and should not contribute as a donor level. Interestingly a calculation of the formation energy in the same work denies the possibility of formation of high isolated $\mathrm{V}_{\mathrm{N}}$ concentrations unless the material is highly p-type.

Here we present a photoluminescence study of autodoped highly conducting AlGaN and GaN layers applying large hydrostatic pressure in search for the possible level of $\mathrm{V}_{\mathrm{N}}$ in the region of 3.0 to $3.8 \mathrm{eV}$. Being a strongly localized defect the energy levels of the $\mathrm{V}_{\mathrm{N}}$ should be independent to a high degree from the energetic position of the band edges. Shifting the band edge by alloying, e.g. in AlGaN, or under hydrostatic pressure would then separate hydrogenic levels from levels of strongly localized defects. Such a behavior has been observed for several defects, e.g. in AlGaAs [14].

\section{Experimental}

Nominally undoped $\mathrm{Al}_{0.15} \mathrm{Ga} 0.85^{\mathrm{N}} / \mathrm{GaN} /$ sapphire heterostructure samples were grown by atmospheric pressure MOCVD using the technique of low temperature AlN buffer layers [15]. Layer thickness was $2 \mu \mathrm{m}(\mathrm{GaN})$ and $1 \mu \mathrm{m}(\mathrm{AlGaN})$. Low temperature $(6 \mathrm{~K}) \mathrm{PL}$ was excited by a $10 \mathrm{~mW}, 325 \mathrm{~nm}$ line of a HeCd laser, dispersed by a $1 \mathrm{~m}$ double monochromator (SPEX) and detected by a UV sensitive photomultiplier tube using lock-in detection. Hydrostatic pressure was applied by means of a Merrill-Bassett type diamond anvil cell using the fluorescence of $\mathrm{R}_{1}$ ruby lines at $6 \mathrm{~K}$ as secondary standard for the pressure determination [16].

\section{Results}

PL of the heterostructure at $0.7 \mathrm{GPa}$ (a) and $3.4 \mathrm{GPa}$ (b) in the vicinity of the bandgaps is presented in Fig. 1. Spectra are given for excitation from the AlGaN layer side (full lines) and the substrate side (dashed lines) (refer to the CB schematic in the inset). Luminescence of the $\mathrm{AlGaN}$ layer is blocked when excited from the substrate side and only a signal from the $\mathrm{GaN}$ 


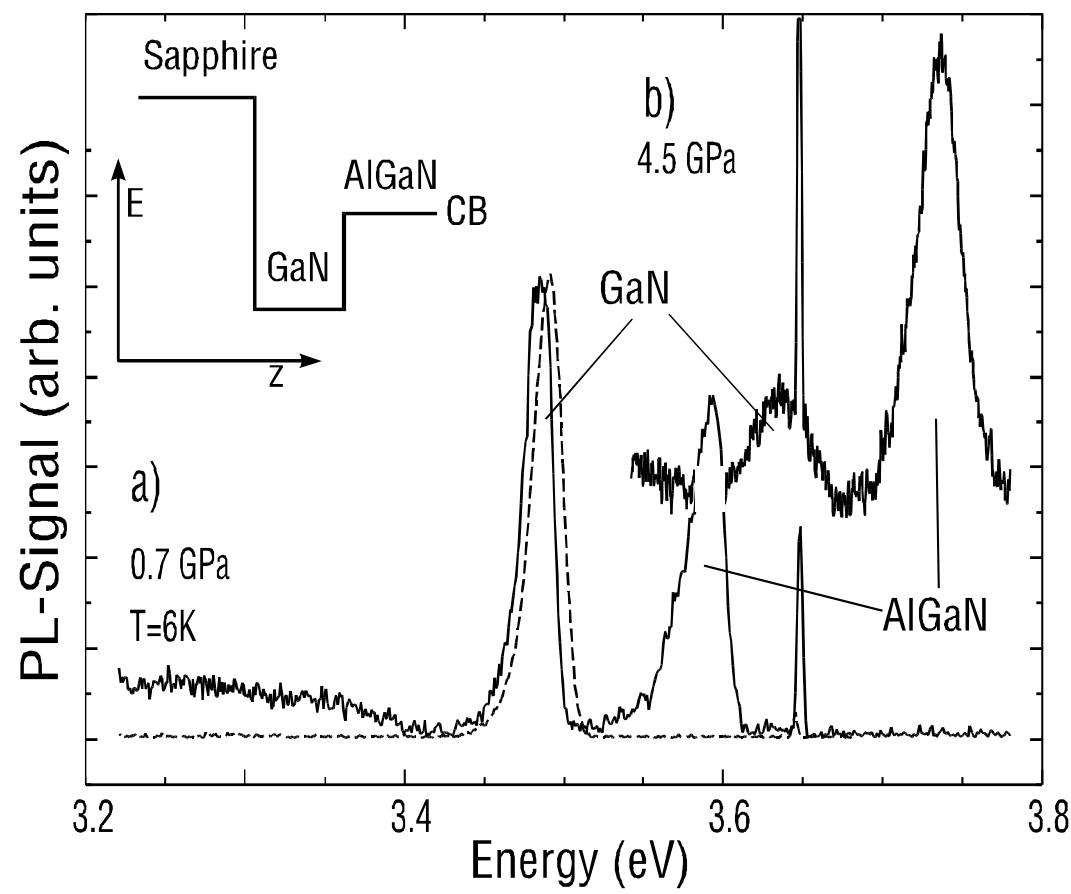

Figure 1: PL excitation from the AlGaN side (full line) and through the substrate (dashed line) at $0.7 \mathrm{GPa}$ (a) and 4.5 $\mathrm{GPa}(b)$. layer is observed. Excitation from the top shows the shallow bound excitons of both of the layers and a defect related contribution on the lower edge of the spectrum. These defects are located in the AlGaN layer, as the luminescence can only be excited through the $\mathrm{AlGaN}$ band edge. There is a small separation of $6.7 \mathrm{meV}(0.7$ $\mathrm{GPa})$ and $8.7 \mathrm{meV}(3.4 \mathrm{GPa})$ in the excitonic luminescence of the $\mathrm{GaN}$ layer that corresponds to the band edge shift due to different bi-axial strain conditions on the lower and the upper GaN interfaces [17]. Both excitons show an asymmetric lineshape with an exponential tail to the low energy side. This is especially obvious on a logarithmic scale (Fig. 3). Besides strain effects this tail width is very sensitive to the presence of a high density of defect states at this energy. In photoluminescence an additional

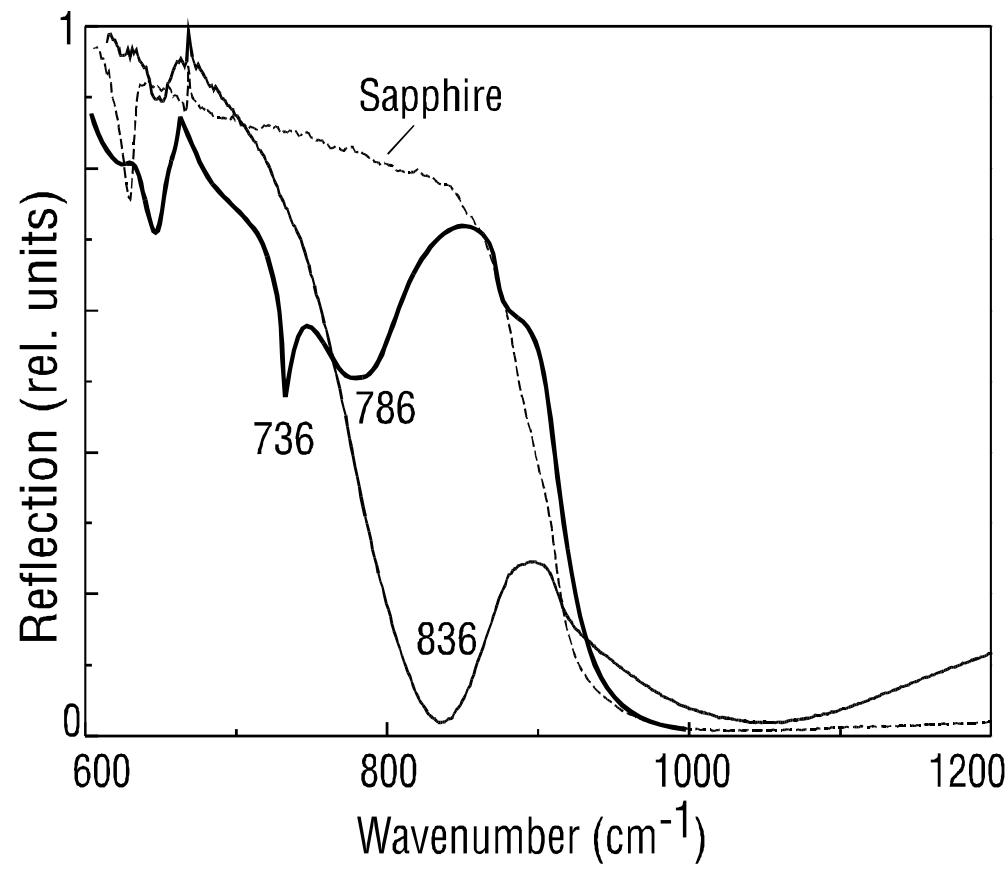

Figure 2: Infrared reflection of the heterostructures. The phonon-plasmon coupled modes reduce the reflection of the sapphire (dashed) reststrahlenband. very narrow line is observed in all spectra at $3.6466 \mathrm{eV}$ and stems from a first order LO-Raman line of the $\mathrm{HeCd}$ laser in the diamonds of the pressure cell.

The high defect and carrier concentration in the individual layers of these films is confirmed by the infrared experiment in Fig. 2. Within the phonon region reflection is dominated by the reststrahlenband of the sapphire substrate with a high frequency edge at about $900 \mathrm{~cm}^{-1}$. The 
layers on top of this mirror-like substrate give rise to pronounced dips at the energies of the LO phononplasmon coupled modes in the individual layers. From the position and the line shape within an oscillator model of the dielectric function the carrier concentration is derived $(\mathrm{N}=$ $\left.1 \times 10^{18}-2 \times 10^{18} \mathrm{~cm}^{-3}\right)$ using parameters of GaN [18].

According to the typical behavior of strongly localized defects a pressure dependence different from the band edges can be expected under hydrostatic pressure. Spectra are obtained from ambient pressure up to 7 $\mathrm{GPa}$. The peak positions of the shallow bound excitons are collected in Fig. 4

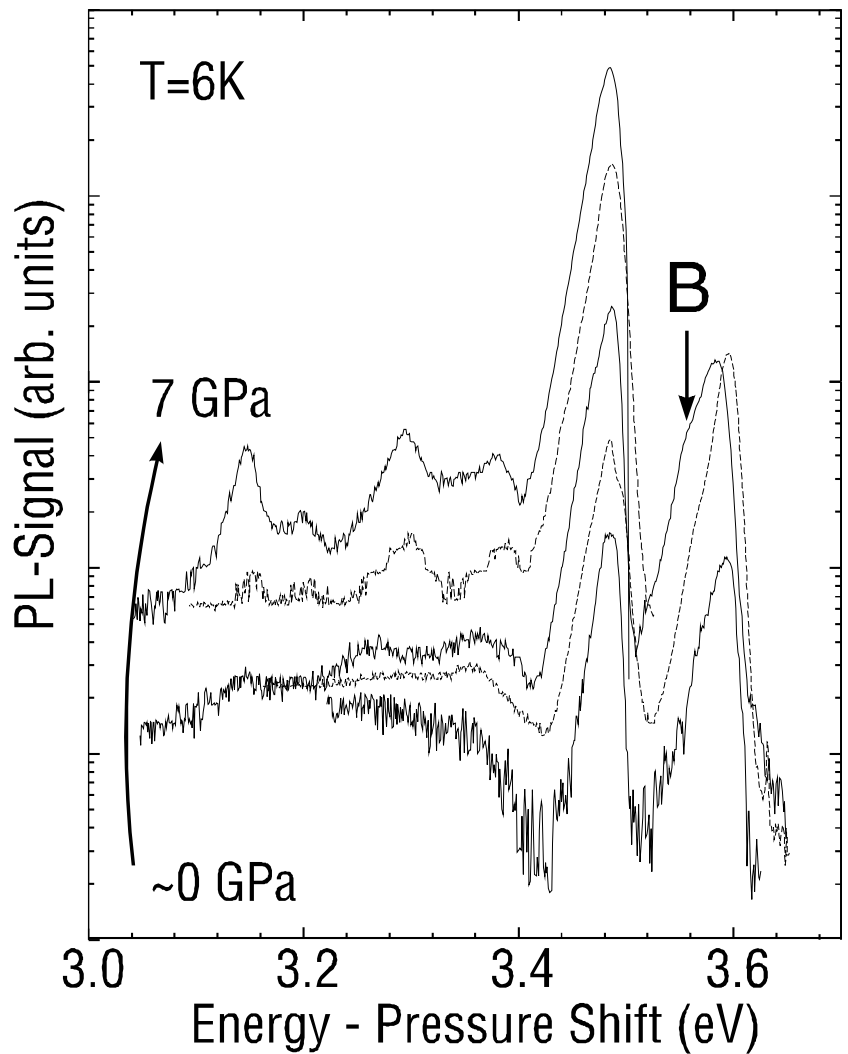

Figure 3: PL on a logarithmic scale versus the energy shifted by the pressure increase of the GaN excitonic gap. (squares $\mathrm{GaN}$, circles $\mathrm{AlGaN}$ ). Within our pressure range we find a linear shift of the exciton position with pressure.

$$
\begin{aligned}
\mathrm{dE} / \mathrm{dp} & =43.7 \pm 1.0 \mathrm{meV} / \mathrm{GPa} \text { in } \mathrm{GaN} \\
& =44.3 \pm 1.4 \mathrm{meV} / \mathrm{GPa} \text { in } \mathrm{Al}_{0.15} \mathrm{Ga}_{0.85} \mathrm{~N}
\end{aligned}
$$

For the ease of interpretation the spectra displayed in Fig. 2 on a logarithmic scale are corrected for the pressure shift of the GaN exciton. We thus realize that all features move parallel to the excitonic lines. The defect related features in the AlGaN layer reveal a donor acceptor transition structure including several phonon replica. At higher pressure they are especially pronounced. The line width of the excitonic transitions shows only little change in the GaN layer (FWHM $\sim 26 \mathrm{meV}$ ), in the AlGaN layer it slightly increases up to $\sim 35 \mathrm{meV}$ at $4.6 \mathrm{GPa}$ (marker B in Fig. 3). From the peak positions (Fig. 4) we derive a binding energy of the donor acceptor transition of $229 \pm 7 \mathrm{meV}$ with respect to the exciton or $\sim 260 \mathrm{meV}$ with respect to the band edge assuming and exciton binding energy of $30 \mathrm{meV}$. Phonon replica appear with energies of 85 to $96 \mathrm{meV}$ in first and second order (see dotted lines in Fig. 4 parallel to excitonic shift). 


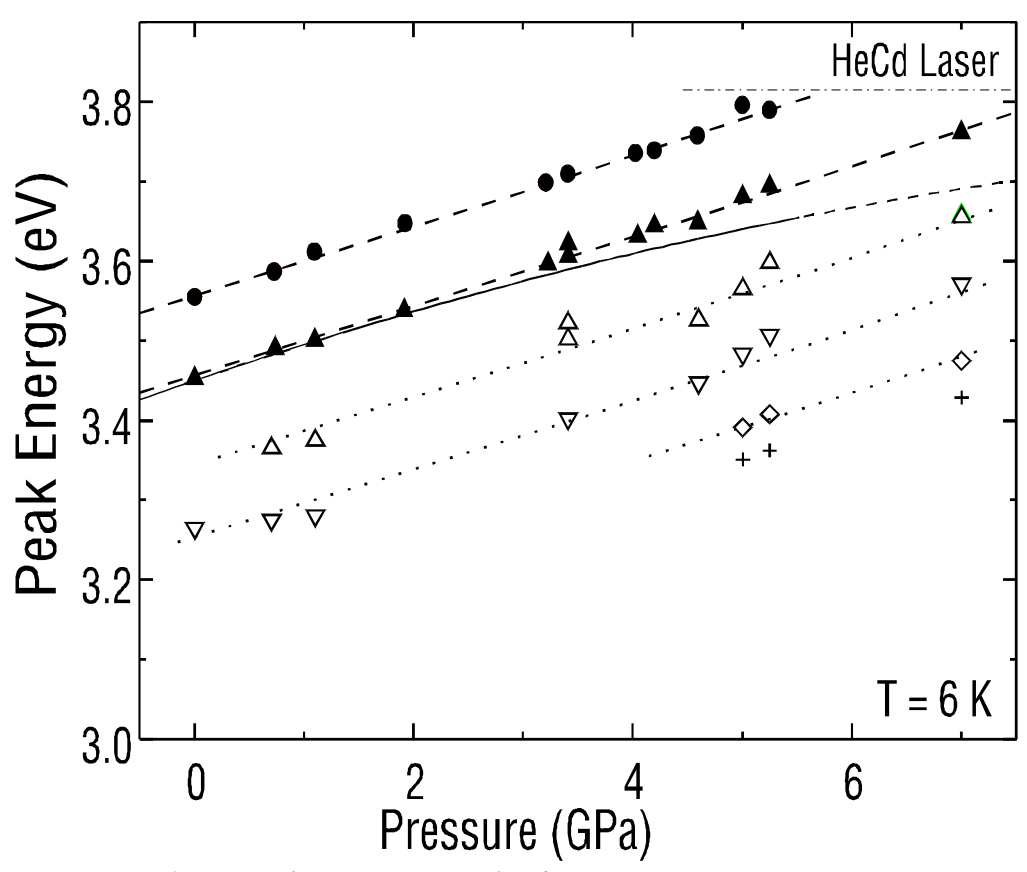

Figure 4: Peakposition of the excitonic transitions in AlGaN (full circles) and GaN (full triangles). The dashed lines are the best linear fit. The defect related transitions are represented by open symbols. The dotted lines are guides for the eye parallel the excitonic shift. The full line is the result of Ref. [19].

clearify this point. Besides this feature we find no indication of strongly localized defects when shifting the excitons from 3.45 to $3.8 \mathrm{eV}$. We furthermore conclude that within the scope of our interpretation no strongly localized levels lie within the range of 3.45 to $3.7 \mathrm{eV}$ above the respective valence band edge in our samples. In addition no strongly localized defect was observed in photoluminescence in the spectral range covered here down to $3.0 \mathrm{eV}$. It should be mentioned that other experiments indicate a crossing of the $\mathrm{CB}$ with a localized level at about $18 \mathrm{GPa}[19]$ respective below $27 \mathrm{GPa}$ [20].

Within the pressure range up to $7 \mathrm{GPa}$ we find a linear dependence of the excitonic bandgap energies in $\mathrm{GaN}$ and $\mathrm{Al}_{0.15} \mathrm{Ga}_{0.85} \mathrm{~N}$. This is in contrast to available data from absorption in thick samples without substrate [21]. Up to pressures of $5.5 \mathrm{GPa}$ a strong sublinear behavior was observed (drawn line in Fig. 4). Our findings might be related to the bi-axial strain present in our thin film structures as manifested in the GaN peak difference when looking from the top or the substrate side. Similar results are obtained on cubic $\mathrm{GaN}$ samples for $\mathrm{p}<=5 \mathrm{GPa}$ [22].

CW acknowledges support by the Deutsche Forschungsgemeinschaft. Work partially supported by US DOE under Contract DE-AC03-76SF00098, and Ministry of Education, Science, and Culture of Japan Contracts 03243107, 06452114 and Hoso Bunka Foundation. 


\section{References}

[1] I. Akasaki, H. Amano, N. Koide, M. Kotaki, K. Manabe, Physica B 185, 428 (1993).

[2] A.S. Barker Jr. and M. Ilegems, Phys. Rev. B 7, 743 (1973).

[3] N. Koide, H. Kato, M. Sassa, S. Yamasaki, K. Manabe, M. Hashimoto, H. Amano, K. Hiramatsu, and I. Akasaki, J. Crystal Growth 115, 639 (1991).

[4] W. Seifert, R. Franzheld, E. Butter, H. Sobotta, V. Riede, Cryst. Res. Technol. 18, 383 (1983).

[5] B.C. Chung, M. Gershenzon, J. Appl. Phys. 72, 651 (1992).

[6] M. Ilegems, H.C. Montgomery, J. Phys. Chem. Solids 34, 885 (1973).

[7] R.J. Molnar, T.D. Moustakas, J. Appl. Phys. 76, 4587, (1994).

[8] R.D. Dupuis (private communication).

[9] D.W. Jenkins, J.D. Dow, Min-Hsiung Tsai, J. of Appl. Phys. 72, 4130 (1992).

[10] P. Boguslawski, E. Briggs, T.A. White, M.G. Wensell, and J. Bernholc, Diamond, SiC and Nitride Wide-Bandgap Semiconductors, eds. C.H. Carter Jr, G. Gildenblat, S. Nakamura and R.J. Nemanich (Mater. Res. Soc. Proc. 339, Pittsburgh, PA 1994) p. 693.

[11] J. Neugebauer and C.G. Van de Walle, Phys. Rev. B 50, 8067 (1994).

[12] H.P. Maruska, J.J. Tietjen, Appl. Phys. Lett. 15, 327 (1969).

[13] J.I. Pankove, in Non-Stoichiometry in Semiconductors. Proc. of Symp. A3 ICAM 91. Edited by: K.J. Bachmann, H.-L. Hwang, C. Schwab, Amsterdam, Netherlands: NorthHolland, 1992. p. 143-53.

[14] M. Mizuta, M. Tachikawa, H. Kukimoto, and S. Minomura, Jpn. J. Appl. Phys. 24, L143 (1985).

[15] Y. Koide, H. Itoh, N. Sawaki, I. Akasaki, M. Hashimoto, J. Electrochem. Soc. 133, 1956 (1986).

[16] H.K. Mao, J. Xu, P.M. Bell, J. Geophys. Research, 91, 673 (1986).

[17] K. Naniwae, S. Itoh, H. Amano, K. Itoh, K. Hiramatsu, and I. Akasaki, J. Crystal Growth, 99, 381 (1990).

[18] C. Wetzel, D. Volm, B.K. Meyer, K. Pressel, S. Nilsson, E.N. Mokhov, P.G. Baranov, Appl. Phys. Lett. 65,1033 (1994).

[19] P. Perlin, H. Teisseyre, T. Suski, M. Leszczynski, I. Grzegory, J. Jun, and S. Porowski, 22nd International Conference on the Physics of Semiconductors, Vancouver, Canada 1994. Ed. D.J. Lockwood (World Scientific Singapore 1995) p. 2383.

[20] C. Wetzel, S. Fischer, W. Walukiewicz, E.E. Haller, P. Perlin, T. Suski, Bulletin Am. Phys. Soc. 40 (1), 416 (1995); C. Wetzel, W. Walukiewicz, E.E. Haller, I. Grzegory, S. Porowski, T. Suski, (unpublished).

[21] P. Perlin, I. Gorczyca, N.E. Christensen, I. Grzegory, H. Teisseyre, and T. Suski, Phys. Rev. B 45, 13307 (1992).

[22] S.J. Hwang, W. Shan, R.J. Hauenstein, J.J. Song, M.-E. Lin, S. Strite, B.N. Sverdlov, H. Morkoç, Appl. Phys. Lett. 64, 2928 (1994). 